\title{
Exact Wave Function of a Time-Dependent Harmonic Oscillator
}

\author{
Tae Jun Park \\ Department of Chemistry, Dongguk Lniversitv, Seoul 100-715, Korea \\ Received November 12,2001
}

Keywords : Time-dependent. Harmonic Oscillator. Time-space. Transformation.

Increasing theoretical attention has been paid to solving exactly time-dependent quantum mechanical problems for their own mathematical interest and for applications. Among these problems. time-dependent harmonic oscillators are widely studied. The most famous method for solving this problem is the quantum invariant operator by Lewis. ${ }^{l}$ Other methods include the propagator and time-space transformation approaches. ${ }^{3}$ These methods usually have been applied to systems in which only the oscillator frequency changes with time. although $\mathrm{Kim}$ et $a I^{4}$ studied an harmonic oscillator whose mass and frequency depend on time

In this work. we have found the exact solution of the Schuödinger equation for an oscillator whose frequency and position vary in time. We have extended the time-space transfomation ${ }^{5}$ and applied it to remove the time-dependence of the position. The time dependency of the frequency is handled by solving an auxiliary differential equation. With this manipulation. the time-dependent Schrödinger equation has been transformed into a time-independent equation whose solutions are easily obtained.

Consider the Schrödinger equation for a time-dependent harmonic oscillator:

$$
i \hbar^{\partial \Psi(x, t)}=-\hbar^{2} \hat{\partial}^{2} \Psi(x, t)+V(x, t)
$$

where $V(x, t)=1 / 2 m \Omega(t)^{2}\left(x-x_{0}(t)\right)^{2}$ and $\Omega(t)$ and $x_{0}(t)$ are the time-dependent frequency and position of the oscillator. To solve eq. (1). a following unitary transfomation is introduced

$$
\Psi(x, t)=\Phi(x, t) e^{i a(n)\left(x-x_{1} ; t\right) t^{2}} .
$$

where $\alpha(t)$ is a function of $t$ and is to be determined later. Substituting into eq. (1) gives

$$
\begin{aligned}
& i \hbar \frac{\partial \Phi}{\partial t}=-\frac{\hbar^{2} \partial^{2} \Phi}{2 m \partial x^{2}}-\frac{2 i \hbar^{2}}{m} \alpha\left(x-x_{0}\right) \frac{\partial \Phi}{\partial x} \\
& +\left[{ }_{2}^{1} m \Omega^{2}+{ }_{m}^{2 \hbar^{2}} \alpha^{2}+\hbar \dot{\alpha}\right]\left(x-x_{0}\right)^{2} \Phi \\
& -2 \hbar \alpha \dot{x}_{0}\left(x-x_{0}\right) \Phi-{ }_{m}^{i \hbar^{2}} \alpha \Phi
\end{aligned}
$$

To remove the last two terms of eq. (3) which are linear and constant in $\left(x-x_{i j}\right)$, we represent $\Phi(x, t)$ as

$$
\Phi(x, t)=\not(x . t) e^{f i x \cdot t !}
$$

where $f(x, t)$ is to be detenmined. In terms of $\phi(x, t)$ and $f(x, t)$. eq (3) can be written as

$$
\begin{aligned}
& i \hbar\left[\begin{array}{c}
\partial \phi \\
\partial t
\end{array}+\frac{\partial f}{\partial t} \phi\right]=-\frac{\hbar^{2} \partial^{2} \phi}{2 m \partial x^{2}}-\left[\begin{array}{l}
\hbar^{2} \partial f \\
m \partial x
\end{array}+{ }_{m}^{2 i \hbar^{2}} \alpha\left(x-x_{0}\right)\right] \begin{array}{l}
\partial \phi \\
\partial x
\end{array} \\
& -\left[\begin{array}{c}
\hbar^{2} \\
2 m
\end{array}\left\{\begin{array}{l}
\partial^{2} f \\
\partial x^{2}
\end{array}+\left(\begin{array}{l}
\partial f \\
\partial x
\end{array}\right)^{2}\right\}+2 \hbar \alpha \dot{x}_{0}\left(x-x_{0}\right)+{ }_{m}^{i \hbar^{2}} \alpha\right. \\
& \left.+{ }_{m}^{2 i \hbar^{2}} \alpha\left(x-x_{0}\right) \frac{\partial f}{\partial x}\right] \phi \\
& +\left[\frac{1}{2} m \Omega^{2}+\frac{2 \hbar^{2}}{m} \alpha^{2}+\hbar \dot{\alpha}\right]\left(x-x_{0}\right)^{2} \phi .
\end{aligned}
$$

Without loss of generality: we can assume $\partial^{2} f / \partial x^{2}=0$. and thus $\partial f / \partial x=g(t)$. Comparing both sides of the equality in eq. (5), we note that the equation is greatly simplified if $f(x, t)$ satisfies the following relation

$$
\frac{\partial f}{\partial t}={ }_{2 m} m\left(\frac{\partial f}{\partial x}\right)^{2}-{ }_{m}^{2 \hbar} \alpha\left(x-x_{0}\right) \frac{\partial f}{\partial x}-{ }_{m}^{\hbar} \alpha+2 i \alpha \dot{x}_{0}\left(x-x_{0}\right)
$$

where we use the fact that $\partial^{2} f / \partial x^{2}$ is zero. We now obtain $f(x, t)$ by integrating eq. (6) with respect to $t$

$$
\begin{aligned}
f(x, t) & =\int^{\prime}\left[\frac{\hbar \hbar}{2 m^{2}} g^{2}-{ }_{m}^{2 \hbar} \alpha\left(x-x_{0}\right) g\right. \\
& \left.-{ }_{m}^{\hbar} \alpha+2 i \alpha \dot{x}_{0}\left(x-x_{0}\right)\right] d t^{\prime} .
\end{aligned}
$$

where $\partial f / \partial x=g(t)$ is used. The function $g(t)$ needs to be determined in order to have $f(x, t)$ explicitly represented in $x_{i j}$ and $\alpha$, and the equation for $g(t)$ is obtained by differentiating eq. (7) with respect to $x$ as.

$$
g=\frac{\partial f}{\partial x}=-\frac{2 \hbar}{m} \int^{t} \alpha g d t^{\prime}+2 i \int^{t} \alpha \dot{x}_{0} d t^{\prime}
$$

The solution of eq. (8) is obtained as.

$$
g(t)=2 i e^{-\frac{2 \hbar}{m} \int^{t} \alpha d t^{\prime}}\left[\int \alpha \dot{x}_{0} e^{\frac{2 \pi}{m} \int^{t} \alpha d t} d t\right] .
$$


Assuning $\alpha(t)=m /(2 \hbar)(i / t)$, eq. (9) may be written in terms of $t(t)$ as follows:

$$
g(t)={ }_{\hbar}^{m} u^{-1} \int^{t} i \dot{x}_{0} d t^{\prime}
$$

Substitution of $g(t)$ into eq. (7) and a simple algebraic manipulation give

$$
f(x, t)=g\left(x-x_{0}\right)-{ }_{2 h}^{i m} \int^{\prime}\left(w^{2}-\dot{x}_{0}^{2}\right) d t^{\prime}-\frac{1}{2} \ln u,
$$

where $g(t)$ is given above and $w(t)$ is defined as.

$$
w(t)=\left(\begin{array}{c}
i m \\
h
\end{array}\right)^{-1} g-\dot{x_{0}} .
$$

The differential equation for $u(t)$ will be obtained later. Using $f(x, t)$ of eq. (11), we can simplify eq. (5) as follows:

$$
\begin{aligned}
i \hbar \frac{\partial \phi}{\partial t} & =-{ }_{2 m}^{2} \partial x^{2} \phi-\left[\hbar^{2} g+{ }_{m}^{2 i \hbar^{2}} \alpha\left(x-x_{0}\right)\right] \begin{array}{l}
\partial \phi \\
\partial x
\end{array} \\
& +\left[{ }_{2}^{1} m \Omega^{2}+{ }_{m}^{2 \hbar^{2}} \alpha^{2}+\hbar \dot{\alpha}\right]\left(x-x_{0}\right)^{2} \phi .
\end{aligned}
$$

For time-space transformation. 5 we introduce the new variables $y=y(x, t)$ and $s=s(t)$, so as to remove $\partial \phi / \partial x$ in eq. (13) and replace the derivatives in $x$ and $t$ with $y$ and $s$ to have

$$
\begin{aligned}
& i \hbar\left[\left(\begin{array}{l}
\partial y \\
\partial t
\end{array}\right) \frac{\partial \phi}{\partial y}+\left(\begin{array}{l}
\partial s \\
\partial t
\end{array}\right) \begin{array}{l}
\partial \phi \\
\partial s
\end{array}\right]=-{ }_{2 m}^{\hbar^{2}}\left[\left(\begin{array}{l}
\partial y \\
\partial x
\end{array}\right)^{2} \frac{\partial \phi^{2}}{\partial y^{2}}+\left(\begin{array}{l}
\partial^{2} y \\
\partial x^{2}
\end{array}\right) \begin{array}{l}
\partial \phi \\
\partial y
\end{array}\right] \\
& -\left[\begin{array}{c}
\hbar^{2} \\
m^{2}
\end{array}\right. \\
& +\left[{ }_{2}^{1} m \Omega^{2}+{ }_{m}^{2 \hbar^{2}} \alpha^{2}+\hbar \dot{\alpha}\right]\left(x-x_{0}\right)^{2} \phi \text {. }
\end{aligned}
$$

Eq. (14) would be reduced to the following:

$$
i \hbar \frac{\partial \phi}{\partial s}=-\frac{\hbar^{2} \partial^{2} \phi}{2 m \frac{\partial y^{2}}{2}}+\left[{ }_{2}^{1} m \Omega^{2}+{ }_{m}^{2 \hbar^{2}} \alpha^{2}+\hbar \dot{\alpha}\right] \dot{s}^{-1}\left(x-x_{0}\right)^{2} \phi
$$

if $\partial y / \partial t$ and $\partial s / \partial t$ satisfy the following coupled differential equations

$$
\begin{gathered}
i \hbar \frac{\partial y}{\partial t}=-\frac{\hbar^{2} \partial \partial^{2}}{2 m \partial x^{2}}-\left[\begin{array}{l}
\hbar^{2} \\
m^{2}
\end{array}+{ }_{m}^{2 i \hbar^{2}} \alpha\left(x-x_{0}\right)\right] \\
\partial s \\
\partial t \\
\partial t \\
\partial x
\end{gathered}
$$

Since $\partial y / \partial x$ is a function of $t$ only as we note from eq. (16b). $\partial^{2} y / \partial x^{2}$ is equal to 0 and the first term of the right hand side of eq. (16a) vanishes. Substituting $\partial y / \partial x=\dot{s}$ and $g(t)$ of eq. (10) into eq. (16a), we get

$$
y(x, t)=u^{-1}\left(x-x_{0}\right)-\int^{t} u^{-1} w d t^{\prime}
$$

where $u(t)$ is given in eq. (12). We can solve the equation for $s(t)$ by inserting $\partial y / \partial x$ obtained from eq. (17) into eq. ( $16 \mathrm{~b})$ :

$$
s(t)=\int^{\prime} u^{-2} d t^{\prime} .
$$

Since $x-x_{i j}$ can be expressed in terms of $y$ we can change eq. (15) into a more tractable form as,

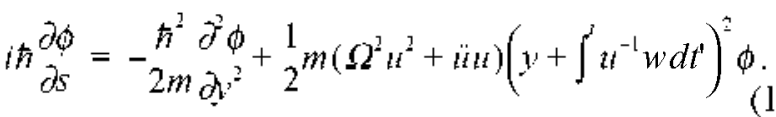

If we transform $y(x, t)$ to $y^{\prime}(x, t)$ such that $y^{\prime}=y+\int^{\prime} u^{-1} u d t^{\prime}$. eq. (19) would become

$$
i \hbar \frac{\partial \phi}{\partial s}=-\frac{\hbar^{2} \partial^{2} \phi}{2 m} \partial y^{\prime 2}+\frac{1}{2} m\left[\Omega^{2} u^{2}+i z u\right] v^{\prime 2} \phi .
$$

where $\partial^{2} / \partial y^{2}$ is replaced with $\partial^{2} / \partial y^{\prime 2}$, because $\partial: \partial x=$ $\partial y \cdot \partial x(\partial \partial y)=\partial y^{\prime} / \partial x\left(\partial / \partial y^{\prime}\right)$ and $\partial y^{\prime} / \partial x=\partial y / \partial x$. Eq. (20) becomes the Schrödinger equation for time-independent harmonic oscillator if the following relation holds:

$$
\ddot{u} u+\Omega^{2} u^{2}=c^{2} .
$$

where $c$ is a constant. Eq. (2l) is the differential equation for $u$ and can be solved when $S(t)$ is given. Then solutions of eq. (20) would be the standard time-independent hamonic oscillator wave functions in $y$ ' and $s$ as

$$
\phi_{n}\left(y^{\prime}, s\right)=N_{n} e^{-\frac{1}{2} \gamma^{2} y^{2}} H_{n}\left(\gamma y^{\prime}\right) e^{-s E_{n} s}
$$

where $N_{n}=\left(\begin{array}{c}\gamma \\ 2^{n} n ! \sqrt{\pi}\end{array}\right)^{\frac{1}{2}} \cdot \gamma=\left(\begin{array}{c}m c \\ \hbar\end{array}\right)^{\frac{1}{2}} \cdot E_{n}=\left(n+\frac{1}{2}\right) \hbar c$. and $H_{n}$ is the Hermite polynomial. From eqs. (2). (4). and (22) along with eqs. (11) and (18). the exact wave function of the nth state is then

$$
\begin{aligned}
& \Psi_{n}(x, t)=\left(2^{n} n !\right)^{-\frac{1}{2}}\left(\begin{array}{c}
m c \\
\pi \hbar
\end{array}\right)^{\frac{1}{4}} u^{-\frac{1}{2}} \exp \left[-\left(n+\frac{1}{2}\right) \hbar c \int^{\prime} u^{-2} d t^{\prime}\right] \\
& \times \exp \left[\begin{array}{l}
m \\
2 \hbar
\end{array}\left(i u^{-1} i-c u^{-2}\right)\left(x-x_{0}\right)^{2}+g\left(x-x_{0}\right)\right. \\
& \left.-{ }_{2 \hbar}^{i m} \int^{3}\left(u^{2}-\dot{x}_{j}^{2}\right) d t^{\prime}\right] \times H_{u}||-u^{-1}\left(x-x_{j}\right) \mid
\end{aligned}
$$

where $g(t)$ and $w(t)$ are given in terms of $u(t)$ as in eqs. (10) and (12). Solution of eq. (21) would thus completely determine $\Psi_{n}(x, t)$ of eq. (23).

Acknowledgment. This work is supported by the Dongguk University research fund. The author dedicates this article to Professor Kyung Hoon Jung.

\section{References}

1. Lewis. Jr.. H. R. J. Math. Phus. $1968,9.1976$.

2. Yeon. K. H.: Kim, D. H.: Um, C. I.; George. T. F.: Pandey, L. N. Phis. Ren: A 1997. 55.4023.

3. Feng. M. Phys Rev . 2001. 64.034101-1

4. Ji. J.Y: Kim. T. K.: Kiml. S. P. Phns Rev: A 1995. 51.4268.

5. Feng, M.; Wang, K. Plys. Lett. A 1995. 197. 135. 